\title{
A Wavelet Kernel-Based Primal Twin Support Vector Machine for Economic Development Prediction
}

\author{
Fang Su${ }^{1}$ and HaiYang Shang ${ }^{2}$ \\ ${ }^{1}$ College of Economics, Lanzhou University of Technology, 287 Langongping Road, Qilihe District, Lanzhou City 730050, China \\ ${ }^{2}$ Lanzhou University of Finance and Economics, Gansu 730050, China \\ Correspondence should be addressed to Fang Su; fangsus@sohu.com
}

Received 17 May 2013; Revised 6 July 2013; Accepted 8 July 2013

Academic Editor: Vishal Bhatnagar

Copyright (c) 2013 F. Su and H. Shang. This is an open access article distributed under the Creative Commons Attribution License, which permits unrestricted use, distribution, and reproduction in any medium, provided the original work is properly cited.

\begin{abstract}
Economic development forecasting allows planners to choose the right strategies for the future. This study is to propose economic development prediction method based on the wavelet kernel-based primal twin support vector machine algorithm. As gross domestic product (GDP) is an important indicator to measure economic development, economic development prediction means GDP prediction in this study. The wavelet kernel-based primal twin support vector machine algorithm can solve two smaller sized quadratic programming problems instead of solving a large one as in the traditional support vector machine algorithm. Economic development data of Anhui province from 1992 to 2009 are used to study the prediction performance of the wavelet kernel-based primal twin support vector machine algorithm. The comparison of mean error of economic development prediction between wavelet kernel-based primal twin support vector machine and traditional support vector machine models trained by the training samples with the 3-5 dimensional input vectors, respectively, is given in this paper. The testing results show that the economic development prediction accuracy of the wavelet kernel-based primal twin support vector machine model is better than that of traditional support vector machine.
\end{abstract}

\section{Introduction}

As policy makers examine the future economic plans for their regions, economic development forecasting allows planners to choose the right strategies for the future $[1,2]$. Gross domestic product (GDP) is an important indicator to measure economic development. Thus, economic development prediction means GDP prediction in this study. Artificial neural networks are the popular prediction algorithms, which have high parallel processing and error tolerance ability [3-5]. Bildirici et al. applied artificial neural networks to economic development prediction, and according to tests of equal forecast accuracy, the results suggest obvious advantages of artificial neural networks compared to regression analysis algorithm [6]. Kim et al. presented early warning system of economic crisis based on artificial neural networks; the experimental results indicated that artificial neural networks can predict economic growth effectively [7]. However, artificial neural networks have the shortcomings of local extremum and overfitting [8-10]. Support vector machine based on the statistical learning theory has already outperformed most other prediction algorithms [11-14]. This study is to propose economic development prediction method based on the wavelet kernel-based primal twin support vector machine algorithm (WPTSVM). The wavelet kernel-based primal twin support vector machine algorithm can solve two smaller sized quadratic programming problems instead of solving a large one as in the traditional support vector machine algorithm. In the wavelet kernel-based primal twin support vector machine, Morlet wavelet function [15-18] can be used as its kernel function.

Economic development data of Anhui province from 1992 to 2009 are used to study the prediction performance of the wavelet kernel-based primal twin support vector machine algorithm. In this experiment, we employ the training samples with different dimensional input vector to train the wavelet kernel-based primal twin support vector machine algorithm. The comparison of the prediction values between the wavelet kernel-based primal twin support vector machine model and traditional support vectors machine 
model trained by the training samples with 3-, 4-, and 5dimensional input vectors, respectively, is given; and the comparison of the prediction error between the wavelet kernel-based primal twin support vector machine model and traditional support vector machine model trained by the training samples with 3-, 4-, and 5-dimensional input vectors, respectively, is given. And the comparison of mean error of economic development prediction between wavelet kernelbased primal twin support vector machine and traditional support vector machine model trained by the training samples with the 3-5 dimensional input vectors, respectively, is given. It can be seen that the economic development prediction accuracy of the wavelet kernel-based primal twin support vector machine model is better than that of traditional support vector machine.

The organization of this paper has been described as follows: wavelet kernel-based primal twin support vector machine has been introduced in Section 2; experimental analysis of economic development prediction method based on the wavelet kernel-based primal twin support vector machine algorithm is described in Section 3; and Section 4 gives the conclusions.

\section{The Proposed Wavelet Kernel-Based Primal Twin Support Vector Machine}

The wavelet kernel-based primal twin support vector machine algorithm can solve two smaller sized quadratic programming problems instead of solving a large one as in the traditional support vector machine algorithm. Given a set of training sets $\left\{\left(x_{i}, y_{i}\right)\right\}_{i=1}^{n} \subset R^{m} \times R$, where $x_{i}$ and $y_{i}$ denote the input vector and corresponding output, the two optimization problems of primal twin support vector machine can be modified as follows.

Minimize

$$
\begin{aligned}
T\left(w_{1}, \xi\right)= & {\left[\frac{1}{2}\left(\left\|w_{1}\right\|_{2}^{2}+b_{1}^{2}\right)+\frac{C_{1}}{2}\right.} \\
& \left.\times\left\|f-\left(\theta(A) w_{1}+e b_{1}\right)\right\|_{2}^{2}+C_{2} e^{\prime} \xi\right]
\end{aligned}
$$

subject to

$$
Y-\left(\theta(A) w_{1}+e b_{1}\right) \geq e \varepsilon_{1}-\xi, \quad \xi \geq 0 .
$$

Minimize

$$
\begin{aligned}
T\left(w_{2}, \eta\right)= & {\left[\frac{1}{2}\left(\left\|w_{2}\right\|_{2}^{2}+b_{2}^{2}\right)+\frac{C_{3}}{2}\right.} \\
& \left.\times\left\|h-\left(\theta(A) w_{2}+e b_{2}\right)\right\|_{2}^{2}+C_{4} e^{\prime} \eta\right]
\end{aligned}
$$

subject to

$$
\left(\theta(A) w_{2}+e b_{2}\right)-Y \geq e \varepsilon_{2}-\eta, \quad \eta \geq 0,
$$

where $C_{i}, i=1,2,3,4$, are the penalty factors; $w_{1}, w_{2}$ denote the weight vector; $b_{1}, b_{2}$ denote bias term; $\xi, \eta$ are the positive slack variables; and $\theta(\cdot)$ is the mapping function.
Then, we can obtain primal twin support vector machine by minimizing the following equation:

$$
\begin{aligned}
F(\alpha)= & {\left[\frac{1}{2}\left(\alpha^{\prime} K \alpha+b_{1}^{2}\right)+\frac{C_{1}}{2}\right.} \\
& \left.\times\left\|\left(K \alpha+e b_{1}\right)-f\right\|_{2}^{2}+C_{2} e^{\prime} l \cdot\left(K \alpha+e b_{1}-f, \mu_{0}\right)\right],
\end{aligned}
$$

where $K=K\left(A, A^{\prime}\right)$ and $u_{0}$ is a given $n$-dimensional vector; and $\alpha$ is the Lagrangian multiplier.

It is well known that primal twin support vector machine with appropriate structure is able to gain excellent nonlinear regression function. In the study, wavelet kernel function can be used to deal with input variables of primal twin support vector machine.

Here, Morlet wavelet function can be selected as the kernel function of the proposed primal twin support vector machine, which can be described as follows:

$$
K\left(x, x^{\prime}\right)=\prod_{i=1}^{m} \cos \left(1.75 \times \frac{x-x^{\prime}}{a_{i}}\right) \exp \left(-\frac{\left\|x-x^{\prime}\right\|^{2}}{2 a_{i}^{2}}\right),
$$

where $a_{i}$ are the coefficients of wavelet function.

It is sufficient to prove the inequality:

$$
F[x](w)=(2 \pi)^{-m / 2} \int \exp (-j(w \cdot x)) k(x) d x \geq 0,
$$

where

$$
k(x)=\prod_{i=1}^{m} \cos \left(\frac{1.75 x}{a_{i}}\right) \exp \left(-\frac{\|x\|^{2}}{2 a_{i}^{2}}\right)
$$

$$
\begin{aligned}
& \int \exp (-j(w \cdot x)) k(x) d x \\
&=\prod_{i=1}^{m} \frac{\left|a_{i}\right| \sqrt{2 \pi}}{2}\left(\exp \left(-\frac{\left(1.75+w_{i} a_{i}\right)^{2}}{2}\right)\right. \\
&\left.\quad+\exp \left(-\frac{\left(1.75-w_{i} a_{i}\right)^{2}}{2}\right)\right) .
\end{aligned}
$$

\section{Experimental Analysis}

Economic development data of Anhui province from 1992 to 2009 [19] are used to study the prediction performance of the wavelet kernel-based primal twin support vector machine 


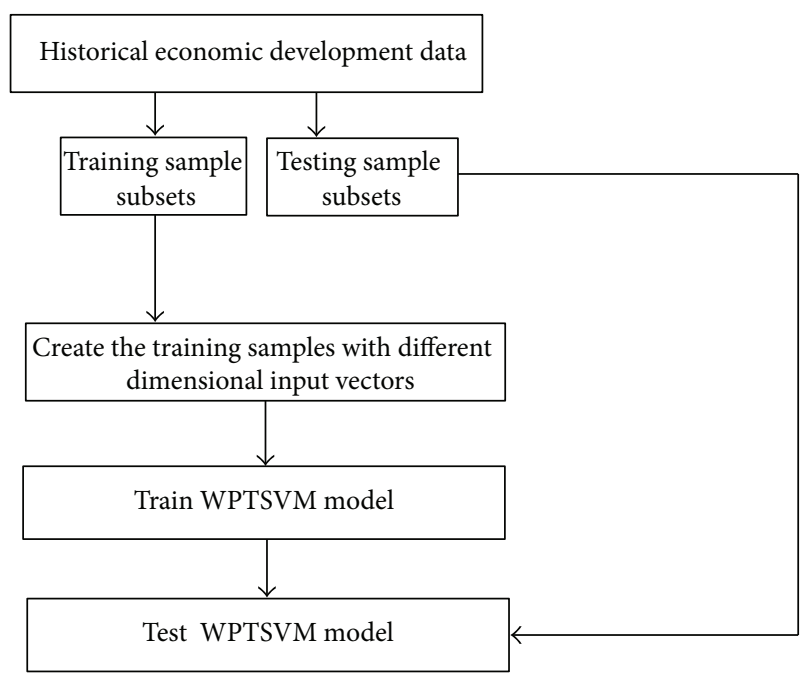

FIGURE 1: The experiment process of the wavelet kernel-based primal twin support vector machine algorithm.

TABLE 1: The comparison of mean error of economic development prediction values between WPTSVM and SVM.

\begin{tabular}{lcc}
\hline $\begin{array}{l}\text { The dimensions of } \\
\text { input vector }\end{array}$ & $\begin{array}{c}\text { Mean error of } \\
\text { PTSVM }\end{array}$ & Mean error of SVM \\
\hline 3 & 0.0253 & 0.0410 \\
4 & 0.0194 & 0.0336 \\
5 & 0.0283 & 0.0462 \\
\hline
\end{tabular}

algorithm. The experiment process of the wavelet kernelbased primal twin support vector machine algorithm can be shown in Figure 1; the training samples are created as follows:

$$
\begin{gathered}
X=\left[\begin{array}{c}
x_{1} \\
x_{2} \\
\cdots \\
x_{n-m}
\end{array}\right]=\left[\begin{array}{cccc}
a_{1} & a_{2} & \cdots & a_{m} \\
a_{2} & a_{3} & \cdots & a_{m+1} \\
\cdots & \cdots & & \cdots \\
a_{n-m} & a_{n-m+1} & \cdots & a_{n-1}
\end{array}\right], \\
Y=\left[\begin{array}{c}
y_{1} \\
y_{2} \\
\cdots \\
y_{n-m}
\end{array}\right]=\left[\begin{array}{c}
a_{1} \\
a_{2} \\
\cdots \\
a_{n-m}
\end{array}\right],
\end{gathered}
$$

where $m$ is the dimension of the input vector.

If the dimension of the input vector is set to 3 , the training samples of this experiment can be described as follows:

$$
\begin{gathered}
X=\left[\begin{array}{l}
x_{1} \\
x_{2} \\
x_{3} \\
\cdots
\end{array}\right]=\left[\begin{array}{lll}
d_{1992} & d_{1993} & d_{1994} \\
d_{1993} & d_{1994} & d_{1995} \\
d_{1994} & d_{1995} & d_{1996} \\
\cdots & \cdots & \cdots
\end{array}\right], \\
Y=\left[\begin{array}{c}
y_{1} \\
y_{2} \\
y_{3} \\
\cdots
\end{array}\right]=\left[\begin{array}{c}
d_{1995} \\
d_{1996} \\
d_{1997} \\
\cdots
\end{array}\right] .
\end{gathered}
$$

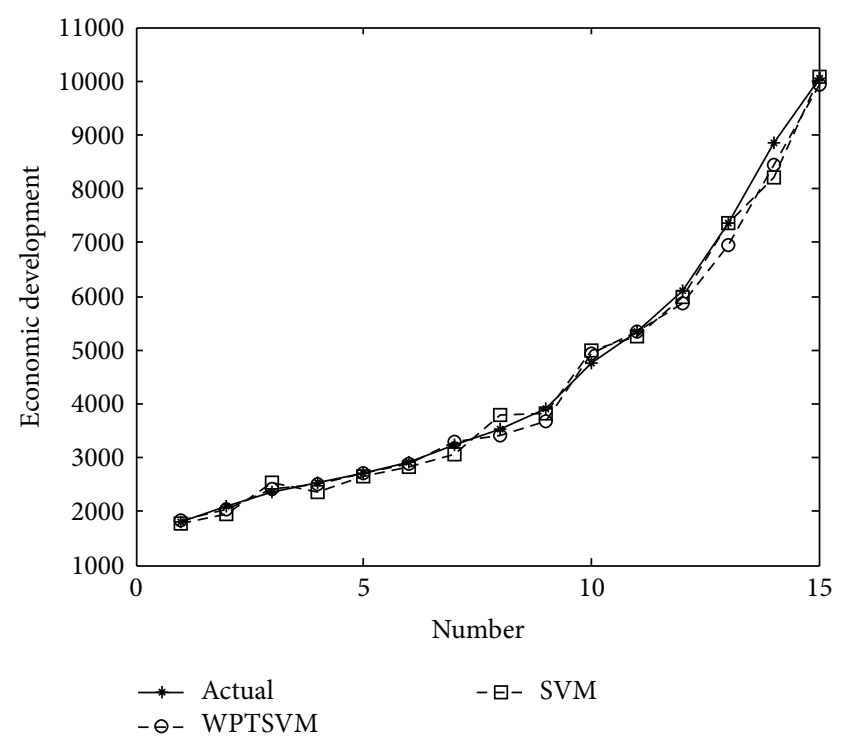

FIGURE 2: The comparison of the prediction values between the WPTSVM model and traditional support vector machine model trained by the training samples with 3-dimensional input vector, respectively.

In this experiment, we employ the training samples with different dimensional input vectors to train the wavelet kernel-based primal twin support vector machine algorithm.

Figure 2 gives the comparison of the prediction values between the wavelet kernel-based primal twin support vector machine model and traditional support vector machine model trained by the training samples with 3-dimensional input vector, respectively; and the comparison of the prediction error between the wavelet kernel-based primal twin support vector machine model and traditional support vector machine model trained by the training samples with 3dimensional input vector, respectively is given in Figure 3. Then, the comparison of the prediction values between the wavelet kernel-based primal twin support vector machine model and traditional support vector machine model trained by the training samples with 4-dimensional input vector, respectively, is given in Figure 4; and Figure 5 gives the comparison of the prediction error between the wavelet kernel-based primal twin support vector machine model and traditional support vector machine model trained by the regression training samples with 4 -dimensional input vector, respectively.

Finally, Figure 6 gives the comparison of the prediction values between the wavelet kernel-based primal twin support vector machine model and traditional support vector machine model trained by the training samples with 5dimensional input vector, respectively; and the comparison of the prediction error between the wavelet kernel-based primal twin support vector machine model and traditional support vector machine model trained by the regression training samples with 5-dimensional input vector, respectively, is given in Figure 7. Table 1 gives the comparison of mean error 


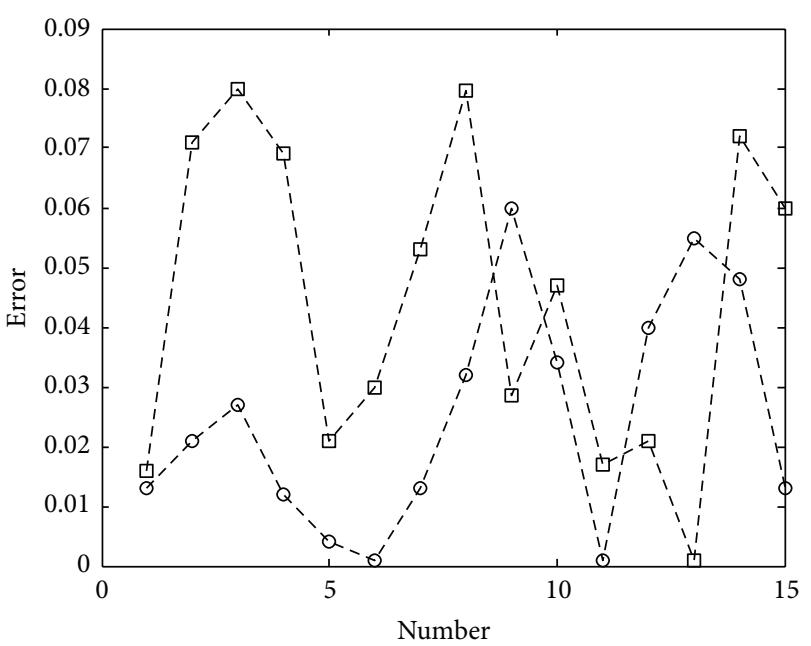

$-\ominus-$ WPTSVM

$-\boxminus-$ SVM

FIGURE 3: The comparison of the prediction error between the WPTSVM model and traditional support vector machine model trained by the training samples with 3 -dimensional input vector, respectively.

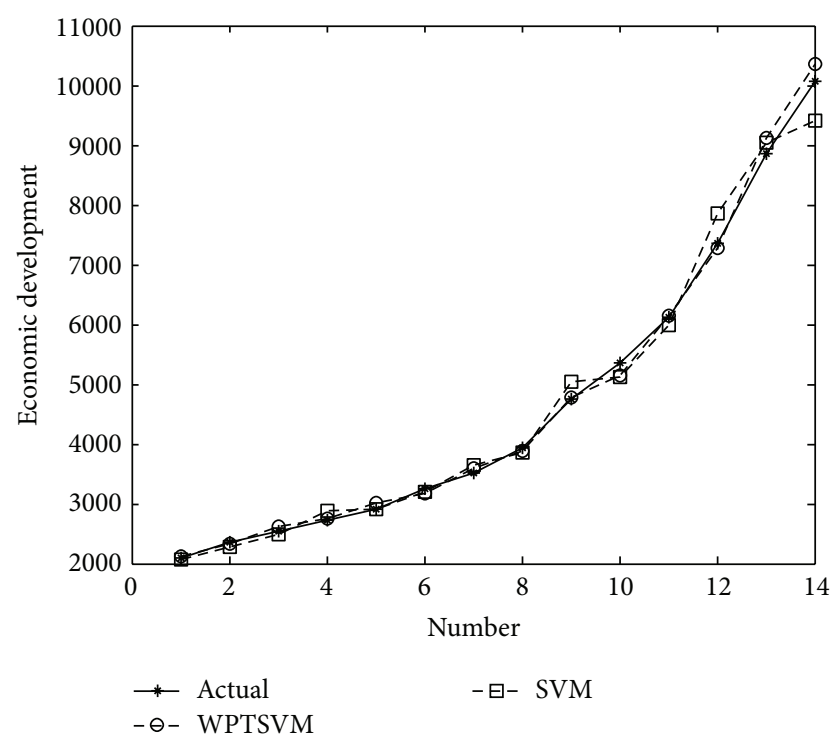

FIGURE 4: The comparison of the prediction values between the WPTSVM model and traditional support vector machine model trained by the training samples with 4-dimensional input vector, respectively.

of economic development prediction between wavelet kernelbased primal twin support vector machine and traditional support vector machine models trained by the training samples with the 3-5-dimensional input vectors, respectively. It can be seen that the economic development prediction accuracy of the wavelet kernel-based primal twin support vector machine model is better than that of traditional support vector machine.

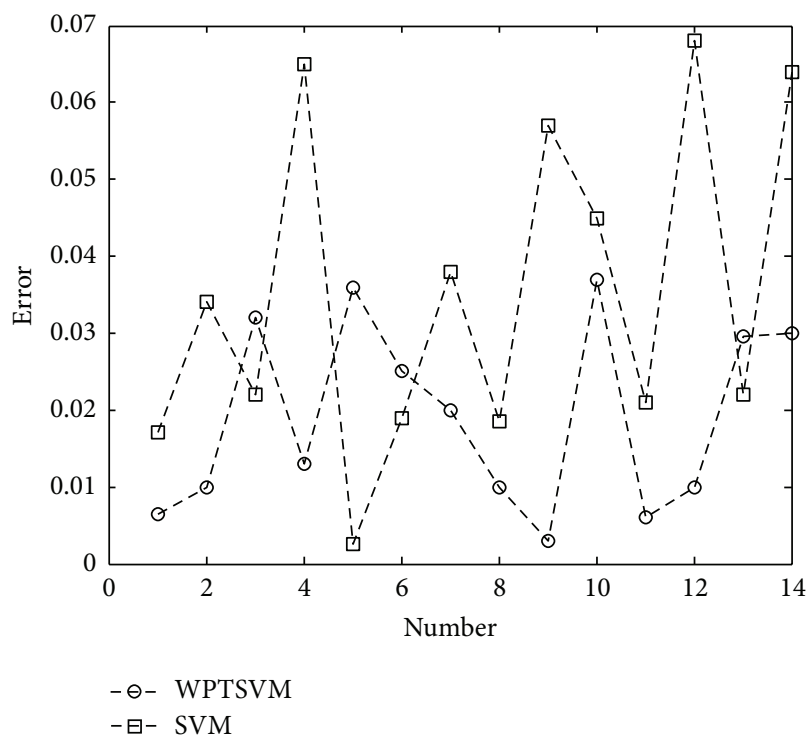

FIgURE 5: The comparison of the prediction error between the WPTSVM model and traditional support vector machine model trained by the regression training samples with 4-dimensional input vector, respectively.

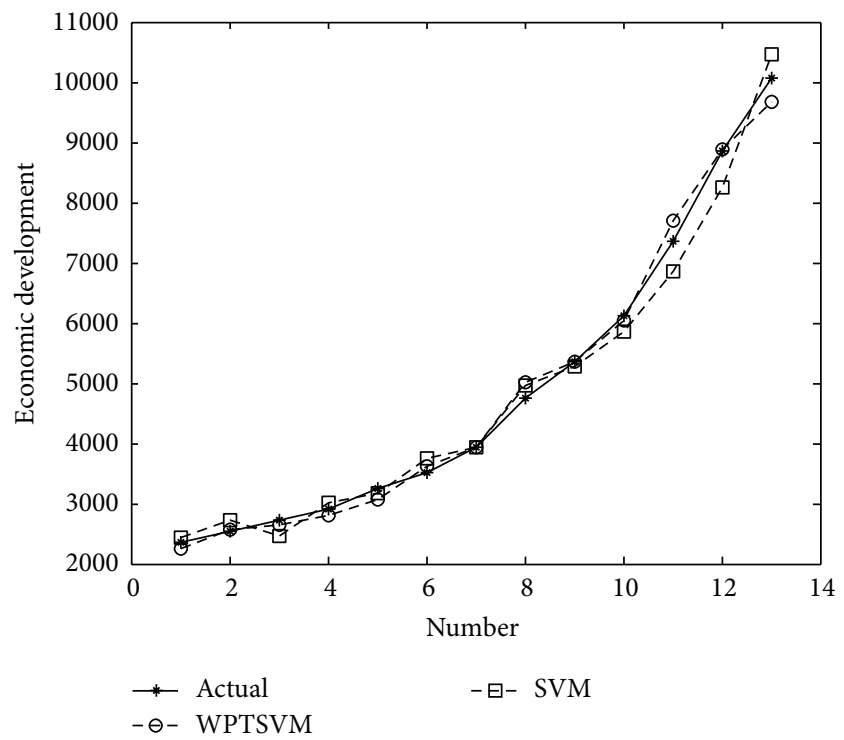

FIgURE 6: The comparison of the prediction values between the WPTSVM model and traditional support vector machine model trained by the training samples with 5 -dimensional input vector, respectively.

\section{Conclusions}

The wavelet kernel-based primal twin support vector machine algorithm is proposed to predict economic development in the paper. The WPTSVM algorithm can solve two smaller sized quadratic programming problems instead of solving a large one as in the traditional support vector machine algorithm. Morlet wavelet function is employed to construct the primal twin support vector machine. 


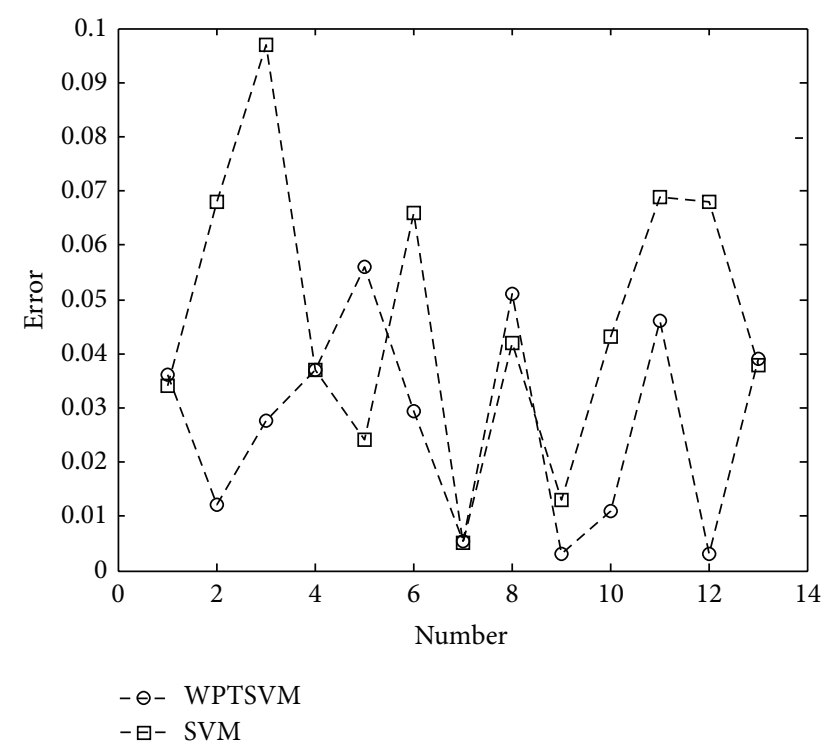

FIGURE 7: The comparison of the prediction error between the WPTSVM model and traditional support vector machine model trained by the regression training samples with 5-dimensional input vector, respectively.

Then, we use multistep prediction mode to indicate the practicability and stability of the wavelet kernel-based primal twin support vector machine algorithm. In this experiment, we employ the training samples with different dimensional input vectors to train the wavelet kernel-based primal twin support vector machine algorithm. The comparison of mean error of economic development prediction between wavelet kernel-based primal twin support vector machine and traditional support vector machine modelstrained by the training samples with the 3-5-dimensional input vectors, respectively is given in this paper. The testing results show that the economic development prediction accuracy of the WPTSVM model is better than that of traditional SVM.

\section{Acknowledgments}

This work is supported by (1) Chinese Natural Science Foundation "Research on the Ecological Compensation Mechanism of the Inner River Basins of the Northwestern China-A Case Study of Shiyang River Basin (Grant no. 41171116)"; (2) General Research on Social Sciences of Ministry of Education of China (Grant no. 12YJAZH110).

\section{References}

[1] S. Anwar and A. Cooray, "Financial development, political rights, civil liberties and economic growth: evidence from South Asia," Economic Modelling, vol. 29, no. 3, pp. 974-981, 2012.

[2] Q. Liang and J. Z. Teng, "Financial development and economic growth: evidence from China," China Economic Review, vol. 17, no. 4, pp. 395-411, 2006.

[3] J. Xu, J. Zhao, Z. Song, and M. Liu, "Prediction of ultimate bearing capacity of Tubular T-joint under fire using artificial neural networks," Safety Science, vol. 50, no. 7, pp. 1495-1501, 2012.

[4] C. L. Wu, K. W. Chau, and C. Fan, "Prediction of rainfall time series using modular artificial neural networks coupled with data-preprocessing techniques," Journal of Hydrology, vol. 389, no. 1-2, pp. 146-167, 2010.

[5] G. D. Wu and S. L. Lo, "Effects of data normalization and inherent-factor on decision of optimal coagulant dosage in water treatment by artificial neural network," Expert Systems with Applications, vol. 37, no. 7, pp. 4974-4983, 2010.

[6] M. Bildirici, Ö. Ö. Ersin, and M. Kökdener, "Genetic structure, consanguineous marriages and economic development: panel cointegration and panel cointegration neural network analyses," Expert Systems with Applications, vol. 38, no. 5, pp. 6153-6163, 2011.

[7] T. Y. Kim, K. J. Oh, I. Sohn, and C. Hwang, "Usefulness of artificial neural networks for early warning system of economic crisis," Expert Systems with Applications, vol. 26, no. 4, pp. 583590, 2004.

[8] M. Xu, T. C. Wong, and K. S. Chin, "Modeling daily patient arrivals at emergency department and quantifying the relative importance of contributing variables using artificial neural network," Decision Support Systems, vol. 54, no. 3, pp. 1488-1498, 2013.

[9] M. Bashiri and A. F. Geranmayeh, "Tuning the parameters of an artificial neural network using central composite design and genetic algorithm," Scientia Iranica, vol. 18, no. 6, pp. 1600-1608, 2011.

[10] J. Shu, Z. Zhang, I. Gonzalez, and R. Karoumi, “The application of a damage detection method using artificial neural network and train-induced vibrations on a simplified railway bridge model," Engineering Structures, vol. 52, pp. 408-421, 2013.

[11] R. Yu and M. Abdel-Aty, "Utilizing support vector machine in real-time crash risk evaluation," Accident Analysis and Prevention, vol. 51, pp. 252-259, 2013.

[12] A. Azadeh, M. Saberi, A. Kazem, V. Ebrahimipour, A. Nourmohammadzadeh, and Z. Saberi, "A flexible algorithm for fault diagnosis in a centrifugal pump with corrupted data and noise based on ANN and support vector machine with hyperparameters optimization," Applied Soft Computing, vol. 13, no. 3, pp. 1478-1485, 2013.

[13] B. Biswal, M. K. Biswal, P. K. Dash, and S. Mishra, "Power quality event characterization using support vector machine and optimization using advanced immune algorithm," Neurocomputing, vol. 103, pp. 75-86, 2013.

[14] L. Han, L. Han, and H. Zhao, "Orthogonal support vector machine for credit scoring," Engineering Applications of Artificial Intelligence, vol. 26, no. 2, pp. 848-862, 2013.

[15] H. Muzhou and H. Xuli, "The multidimensional function approximation based on constructive wavelet RBF neural network," Applied Soft Computing Journal, vol. 11, no. 2, pp. 21732177, 2011.

[16] J. Rafiee, M. A. Rafiee, N. Prause, and M. P. Schoen, "Wavelet basis functions in biomedical signal processing," Expert Systems with Applications, vol. 38, no. 5, pp. 6190-6201, 2011.

[17] R. C. Guido, "A note on a practical relationship between filter coefficients and scaling and wavelet functions of discrete wavelet transforms," Applied Mathematics Letters, vol. 24, no. 7, pp. 1257-1259, 2011. 
[18] J. Rafiee, M. A. Rafiee, and P. W. Tse, "Application of mother wavelet functions for automatic gear and bearing fault diagnosis," Expert Systems with Applications, vol. 37, no. 6, pp. 45684579, 2010.

[19] B. Liu and G. Y. Yang, "Forecasting the economic development of Anhui based on combination forecasting method," Journal of West Anhui University, vol. 27, no. 5, pp. 48-51, 2011 (Chinese). 


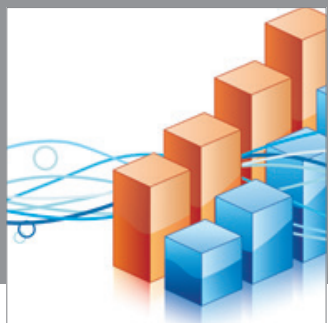

Advances in

Operations Research

mansans

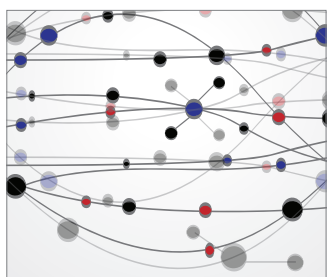

The Scientific World Journal
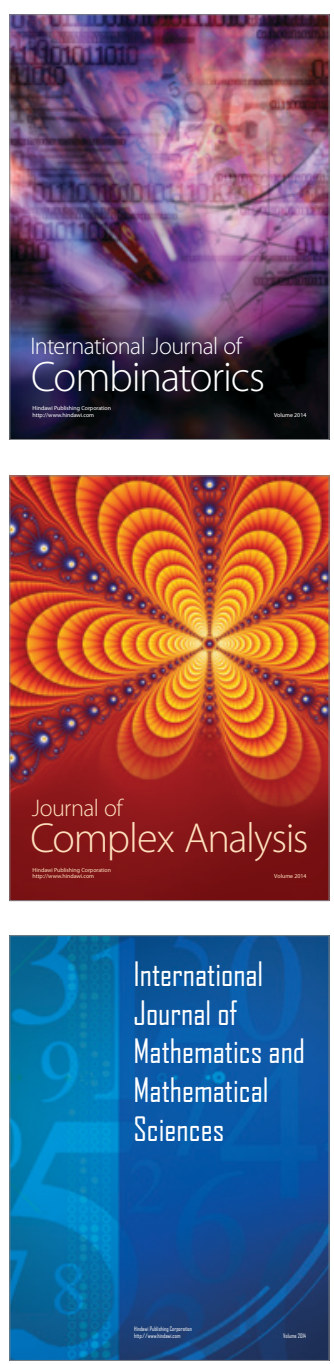
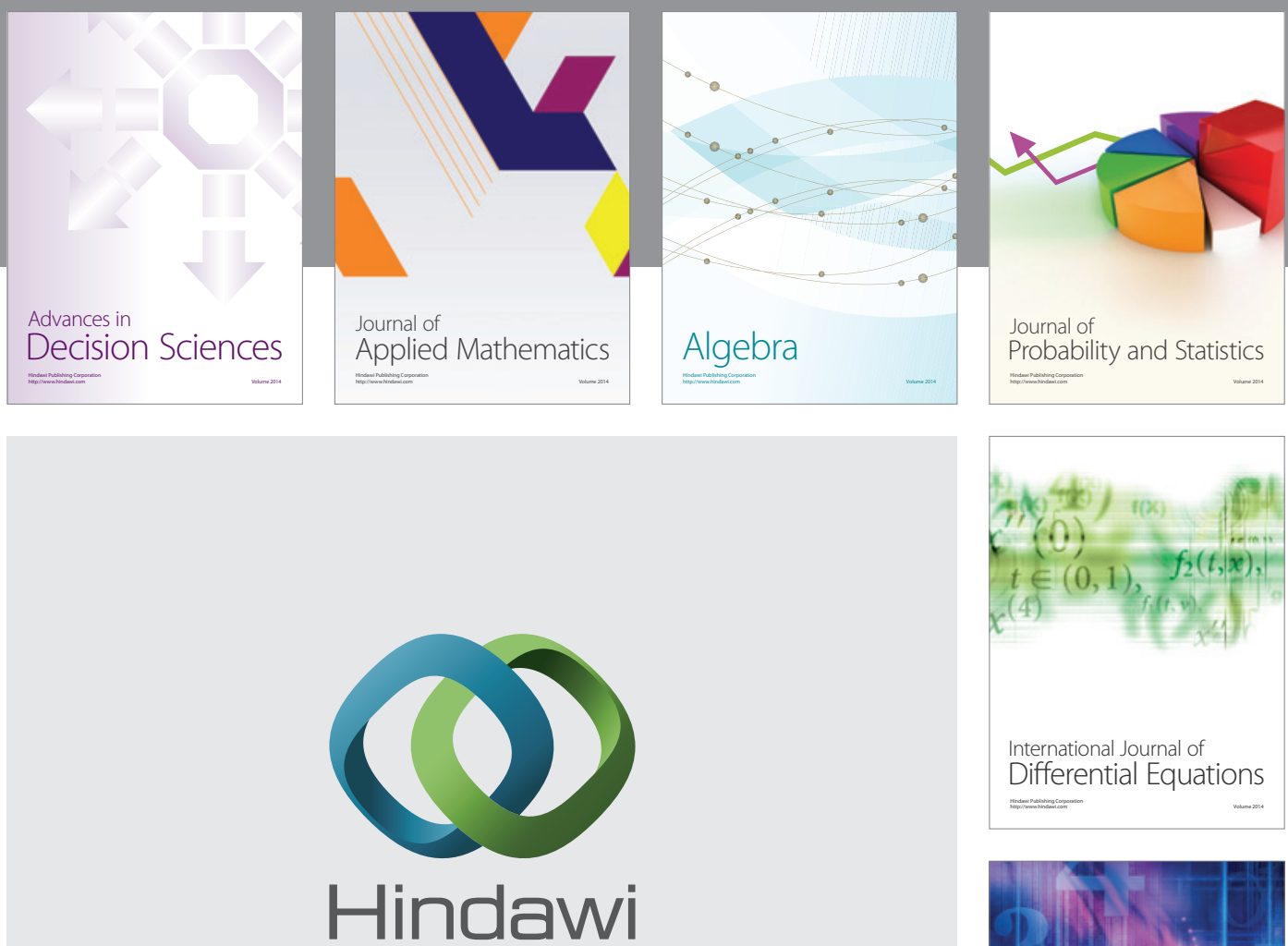

Submit your manuscripts at http://www.hindawi.com
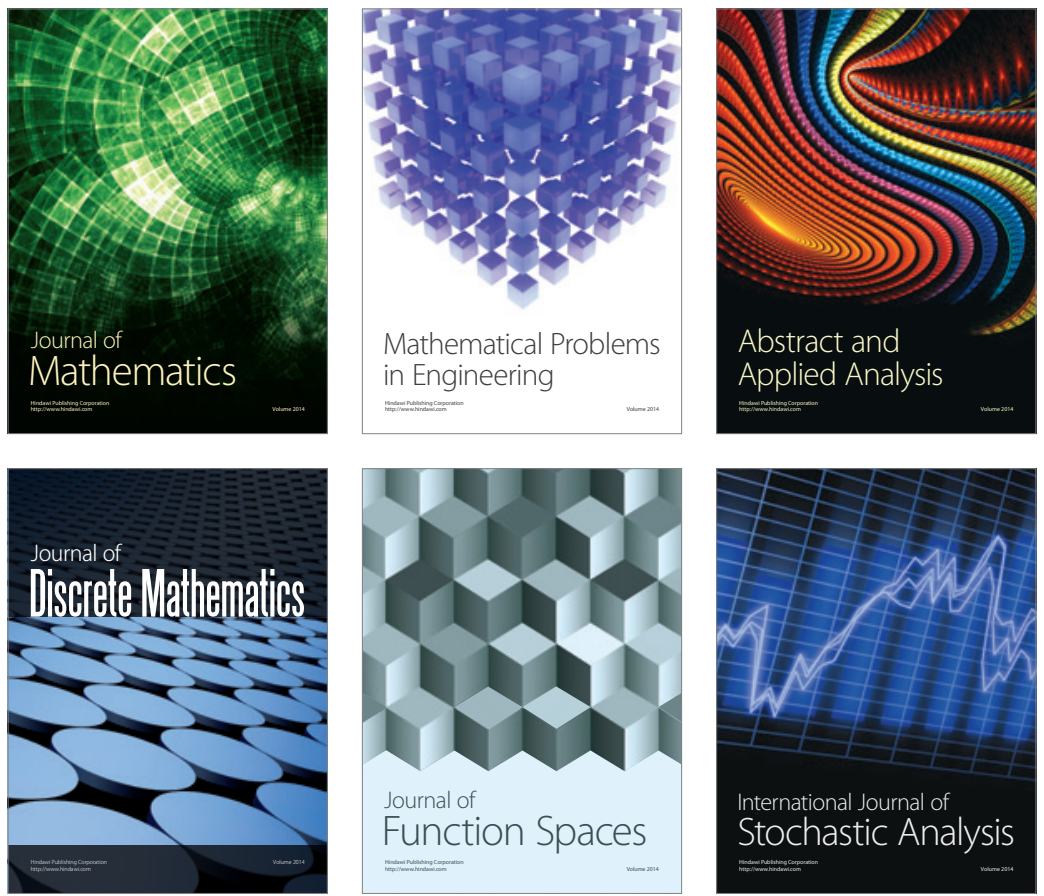

Journal of

Function Spaces

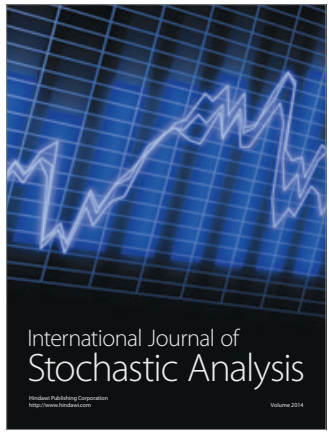

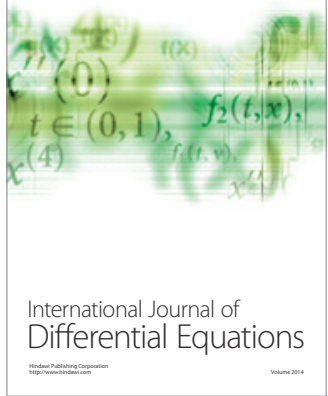
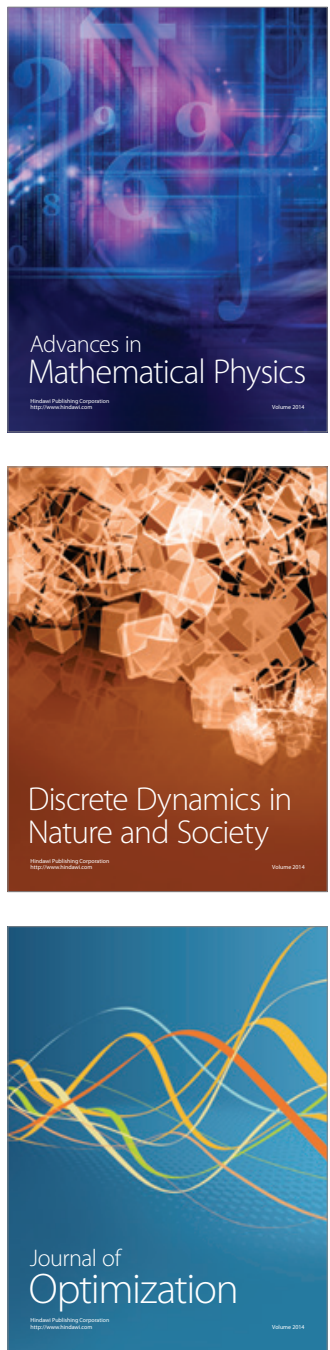